\title{
Probabilistic Graphical Models for Climate Data Analysis
}

\author{
Arindam Banerjee \\ banerjee@,cs.umn.edu
}

Dept of Computer Science \& Engineering

University of Minnesota, Twin Cities

Climate Knowledge Discovery Workshop

November 13, 2011 


\section{Graphical Models}

- Graphical models

- Dependencies between (random) variables, avoid I.I.D. assumptions

- Closer to reality, learning/inference is much more difficult

- Basic nomenclature

- Node $=$ Random Variable, Edge $=$ Statistical Dependency

- Directed Graphs

- A directed graph between random variables

- Example: Bayesian networks, Hidden Markov Models

- Joint distribution is a product of $\mathrm{P}$ (child|parents)

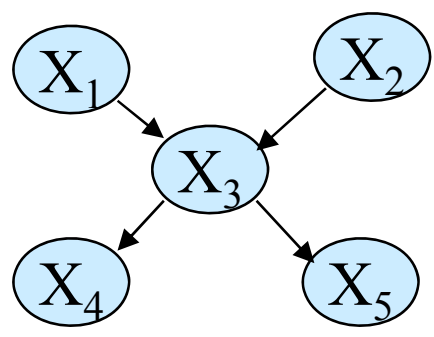

- Undirected Graphs

- An undirected graph between random variables

- Example: Markov/Conditional random fields

- Joint distribution in terms of potential functions

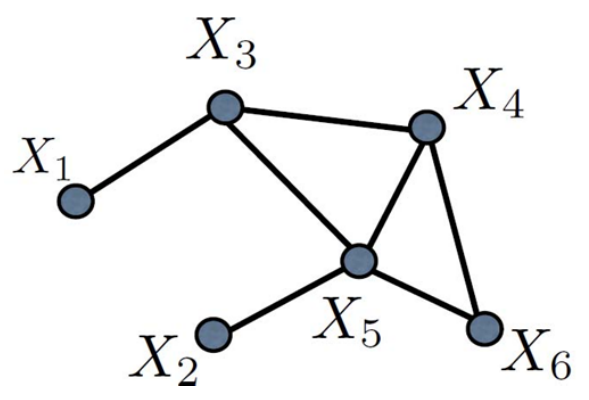




\section{Example: Burglary Network}

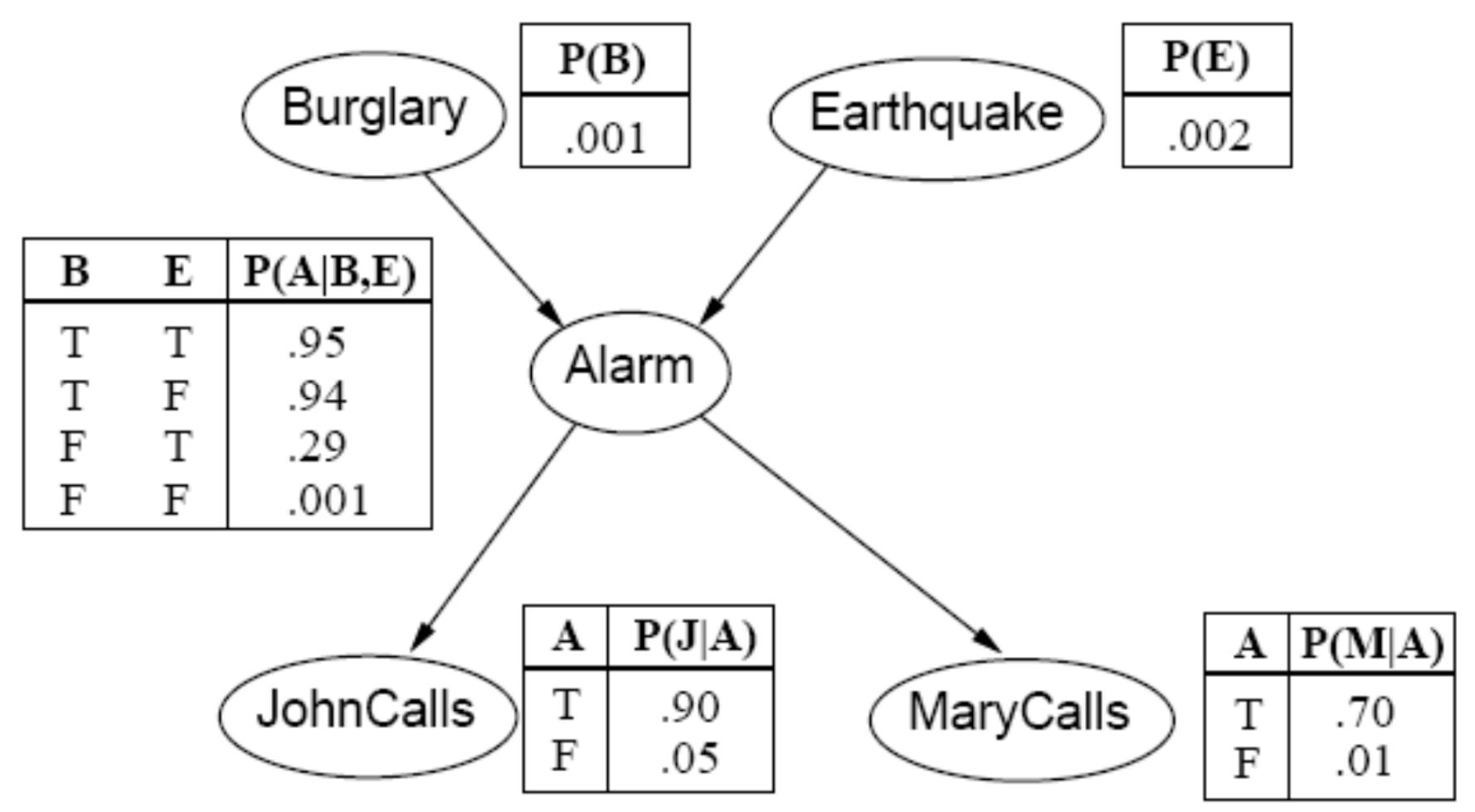




\section{Latent Variable Models}
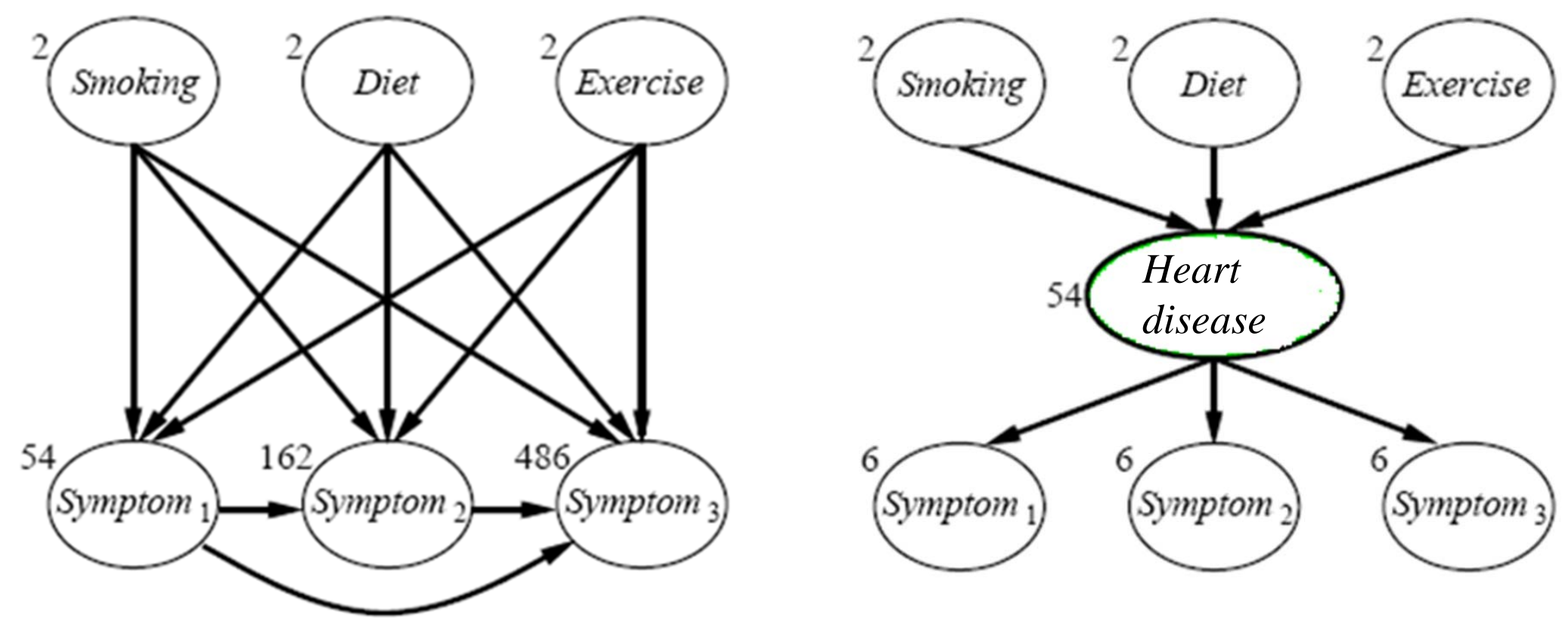

- Bayesian network with hidden variables

- Semantically more accurate, less parameters 


\section{Key Problems}

- Structure Learning

o Given: Samples

o Problem: Learn the Structure

- Parameter Estimation

o Given: Samples and Structure

o Problem: Estimate Parameters

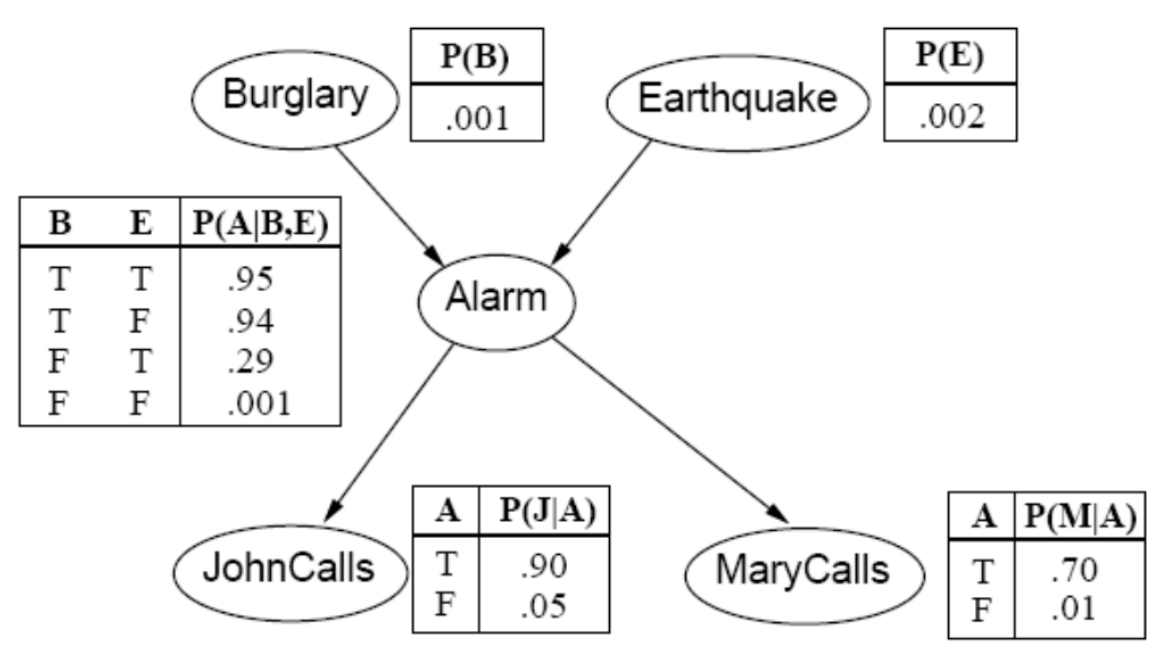

- Inference:

o Given: Structure, Parameters, and some variables (part of a Sample)

o Problem: Find other variables (part of a Sample) 


\section{Abrupt Changes in Climate}

- Significant Droughts

- Persistent over space and time

- Catastrophic consequences

- Examples:

- Late 1960s Sahel drought

- 1930s North American Dust Bowl
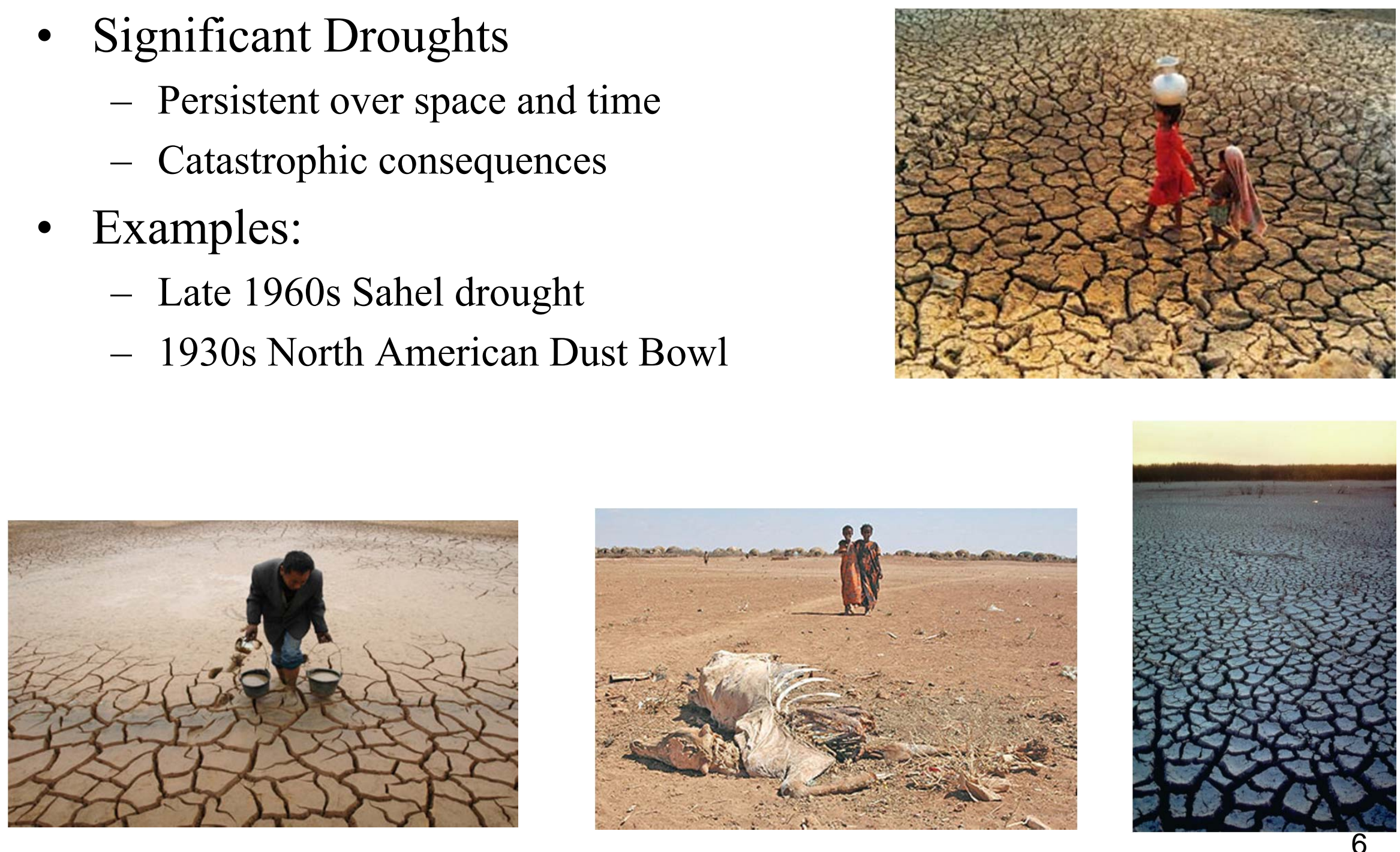


\section{CRU Dataset}

- Monthly precipitation dataset from 1901-2006

- 0.5 latitude x 0.5 longitude

- Precipitation over land only (67420 locations)

- Convert to a yearly dataset to eliminate the monthly variance

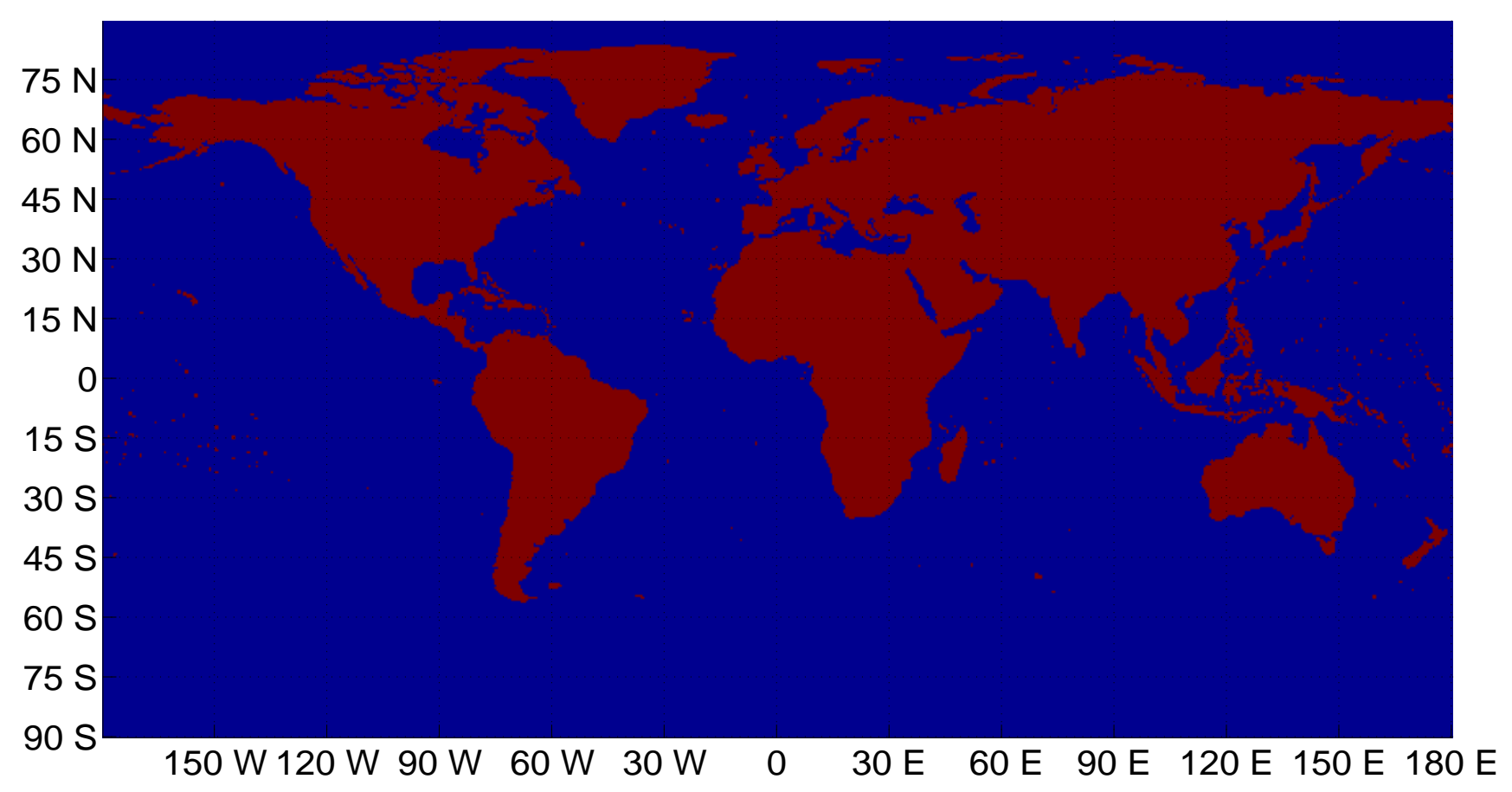




\section{Markov Random Fields (MRF)}

- Undirected graph $\mathrm{G}=(\mathrm{V}, \mathrm{E})$

- Each node takes discrete values from $\mathcal{X}=\{1, \ldots, k\}$

- Node potential $f_{u}\left(x_{u}\right)$

- Edge potential $f_{u v}\left(x_{u}, x_{v}\right)$

- Joint distribution over all variables

$P(x) \propto \exp \left\{\sum_{u \in V} f_{u}\left(x_{u}\right)+\sum_{(u, v) \in E} f_{u v}\left(x_{u}, x_{v}\right)\right\}$

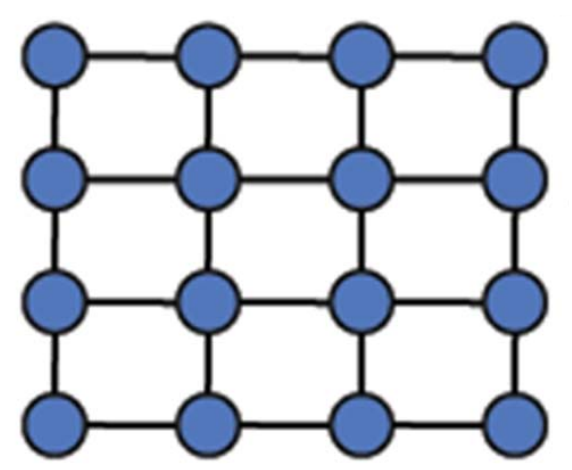

- MAP inference problem

$$
x^{*} \in \operatorname{argmax}_{x \in \mathcal{X}^{n}}\left\{\sum_{u \in V} f_{u}\left(x_{u}\right)+\sum_{(u, v) \in E} f_{u v}\left(x_{u}, x_{v}\right)\right\}
$$




\section{Drought Detection with MAP Inference}

- Model dependencies using a 4-nearest neighbor grid

- Replicate grid over time

- Each node can be 0 (normal) or 1 (dry)

- Toy example:

- $\mathrm{m}=3, \mathrm{n}=4, \mathrm{~N}=12, \mathrm{~T}=5$

- Total \# States: $2^{60}=1.1529 \times 10^{18}$

- CRU data:

- $\mathrm{m}=720, \mathrm{n}=360, \mathrm{~N}=67,420, \mathrm{~T}=106$

- Total \# States: $2^{7,146,520}>10^{2,382,200}$

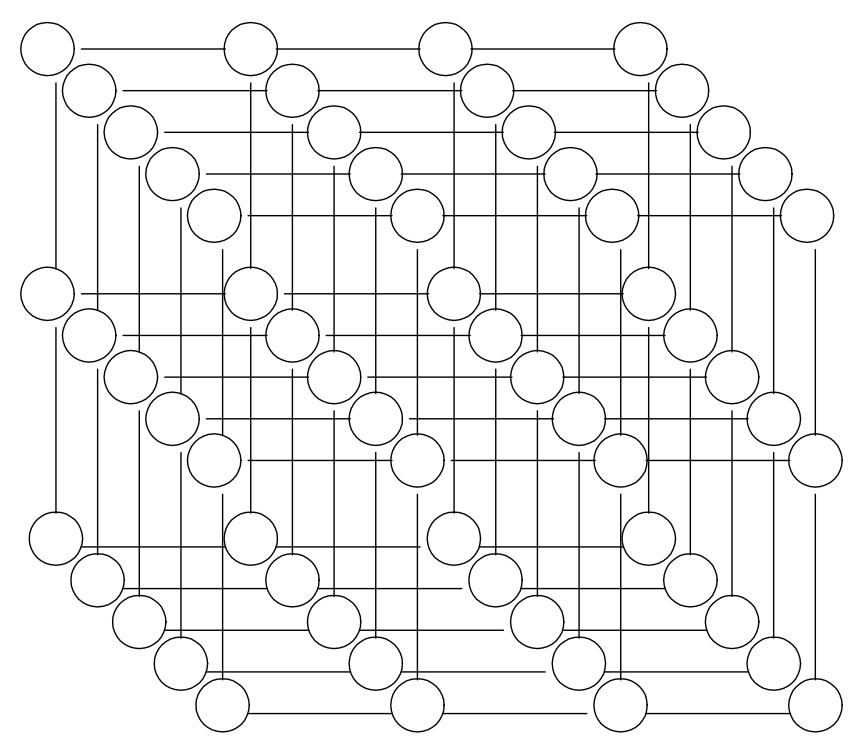

- Drought Detection

- MAP inference: Integer program with millions of variables

- Linear Programming (LP) relaxation

- Post-process the MAP states to identify significant droughts 


\section{Pre-Processing}

- Arid and semi-arid regions are more likely to have droughts

- Calculate the median precipitation for each location

- Cluster the medians

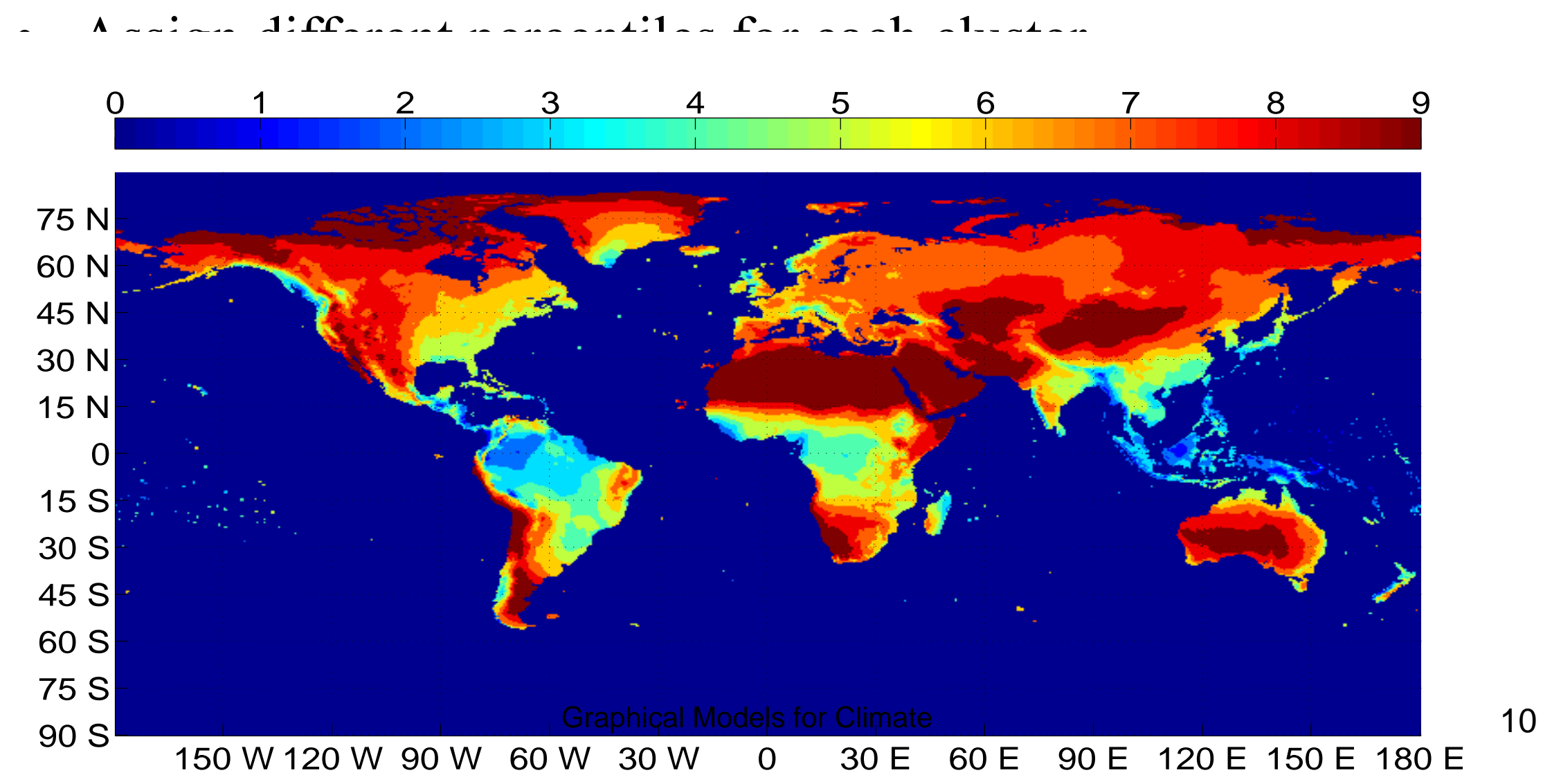




\section{Results: Droughts starting in $1920-30$ s}

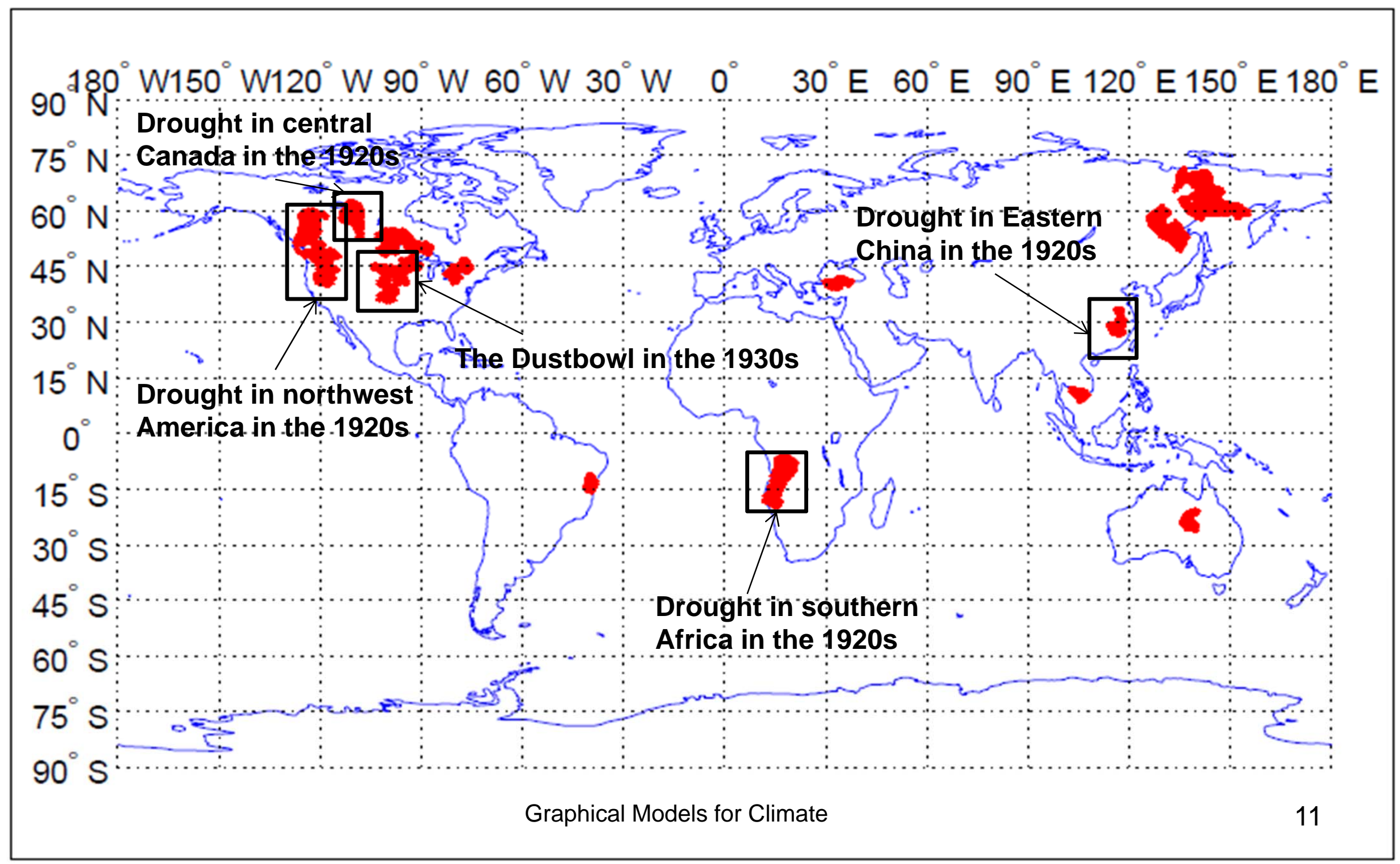




\section{Results: Droughts starting in 1960-70s}

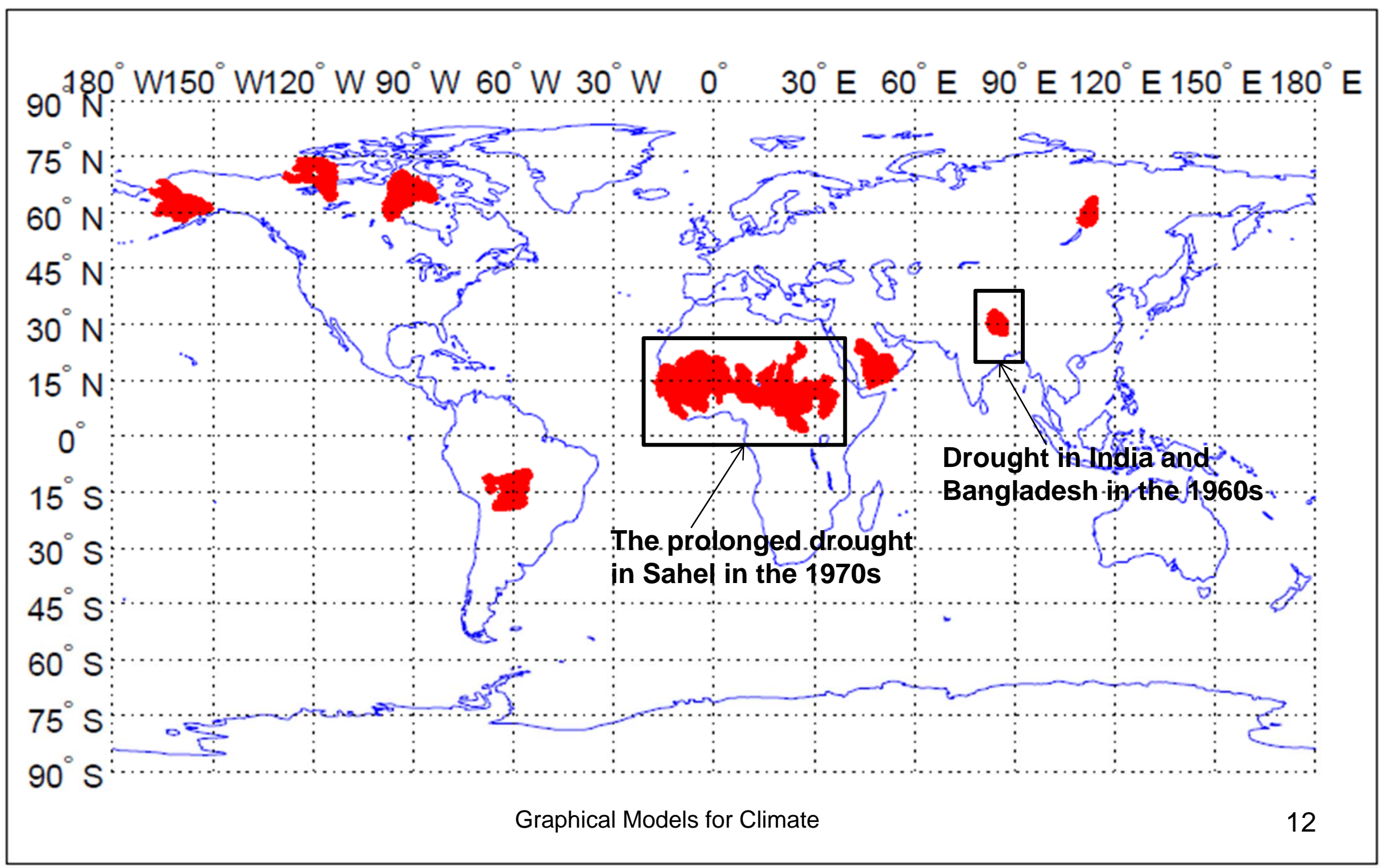




\section{Significant Droughts: 1901-2006}

\section{1}

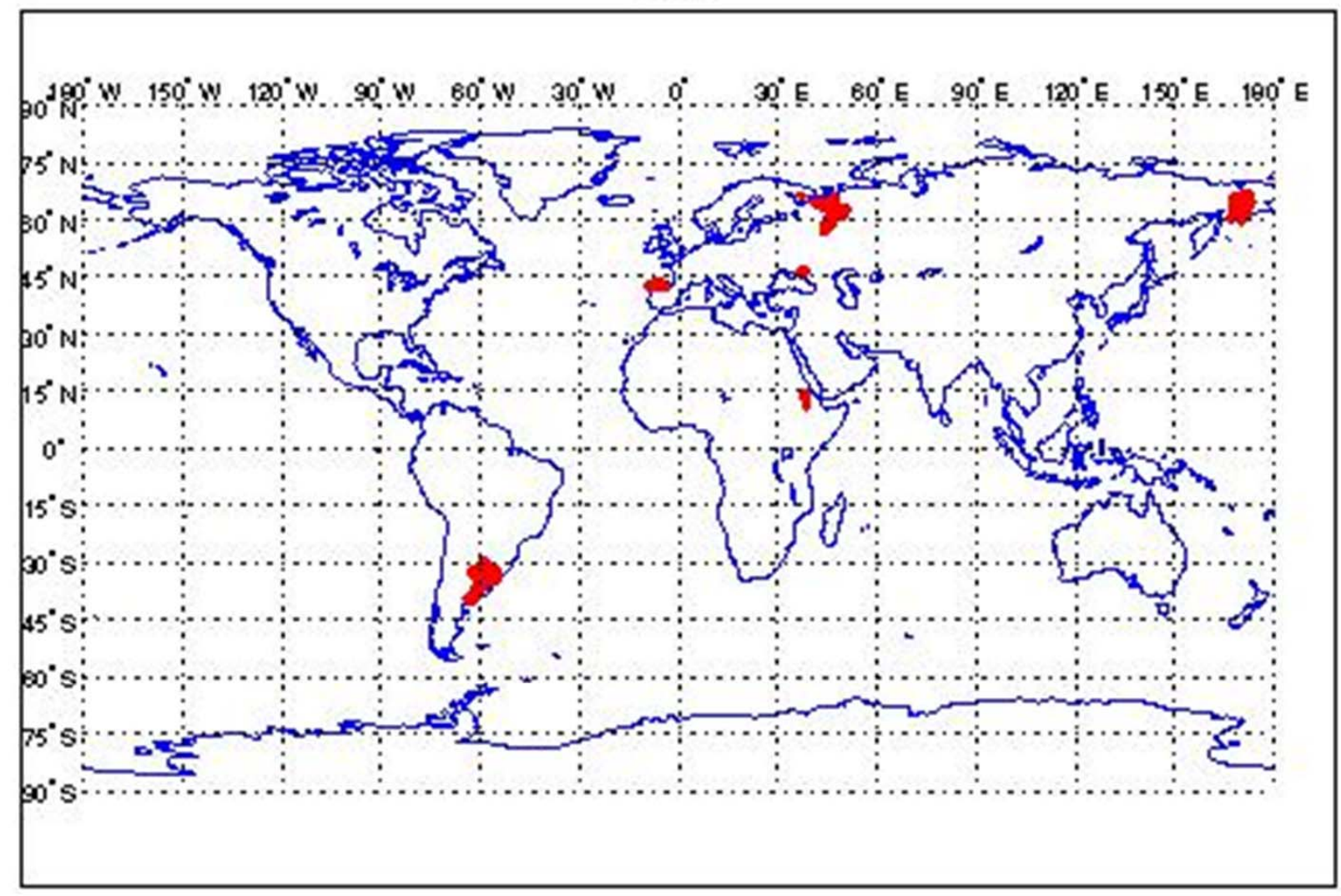




\section{Land Variable Regression}

- Land-Sea variable interactions

- How do sea variables affect land variables?

- Are proximal locations important?

- Are there long range spatial dependencies (tele-connections)?

- NCEP/NCAR Reanalysis 1: monthly means for 1948-2010

- Covariates: Temperature, Sea Level Pressure, Precipitation, Relative Humidity, Horizontal Wind Speed Vertical Wind Speed

- Response: Temperature, Precipitation at 9 locations

- High-dimensional Regression

- Dimension $p=L m, L$ locations, $m$ variables/location

- Two considerations:

- Not all locations are relevant

- Even if a location is relevant, not all variables are relevant 


\section{Land Regions for Prediction: Temp, Precip}

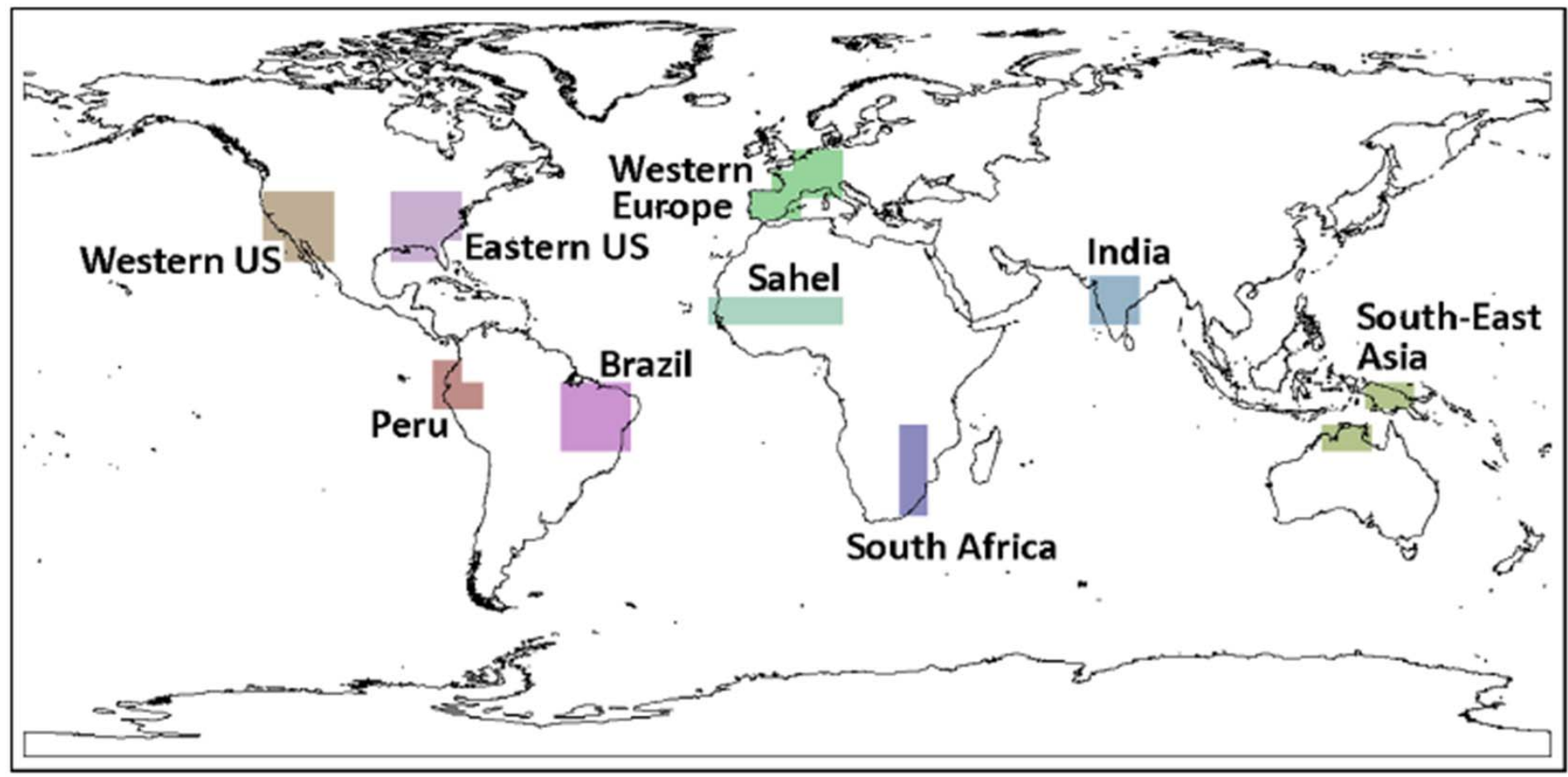

Land regions chosen for prediction 


\section{Dataset and Assumptions}

- NCEP/NCAR Reanalysis 1: monthly means for 1948-2010

- Coarsened to $10^{\circ} \times 10^{\circ}$ spatial resolution

- Anomaly series computed from data

- Training Set : 1948-1997

- Test Set : 1998-2007

- Monthly means

- Consider dependencies over Space

- Considered independent over Time 


\section{Ordinary Least Squares}

- Naive method: Use ordinary least squares (OLS) :

$$
\hat{\theta}:=\underset{\theta \in \mathbb{R}^{m L}}{\operatorname{argmin}}\left\{\frac{1}{n}\|y-X \theta\|_{2}^{2}\right\}
$$

- For $n<m L$, OLS estimate non-unique

- In general, $y$ depends on all $m L$ covariates : complex model

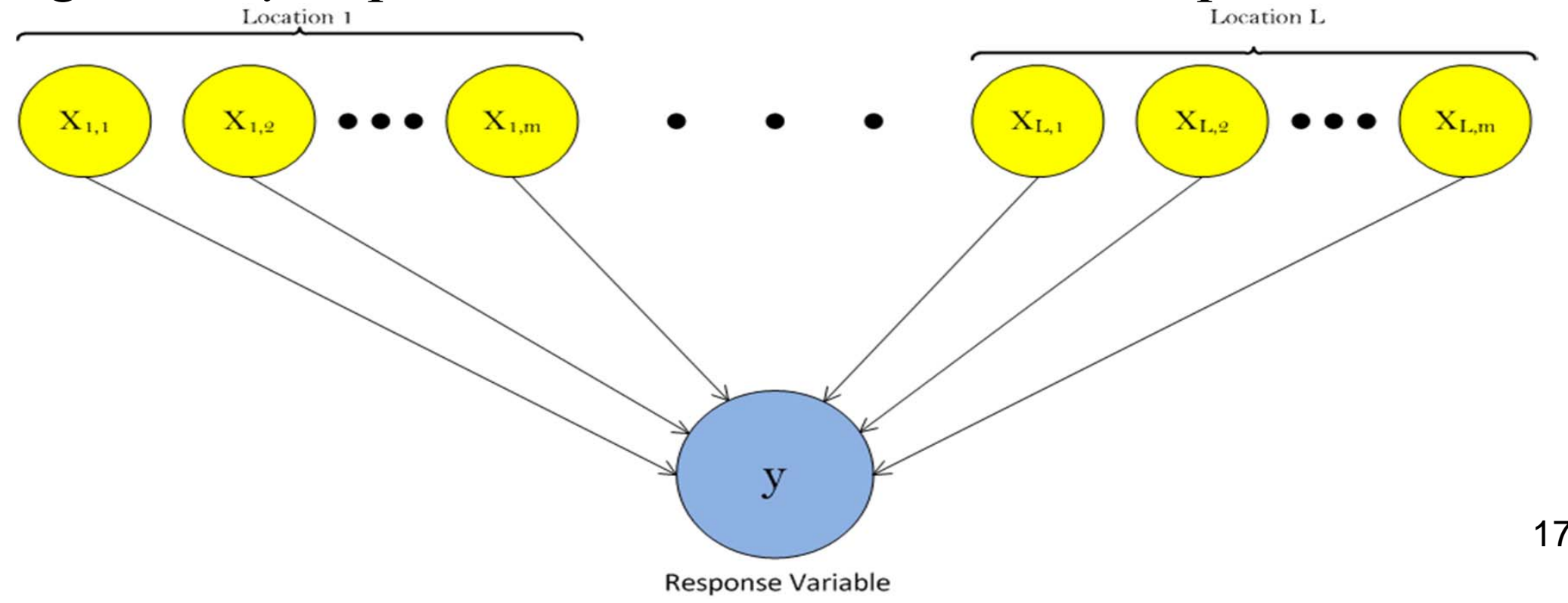




\section{Sparse Regression}

- Add sparsity inducing norm regularizer to OLS

- Examples: $L_{1}$ norm, $L_{1} / L_{q}$ mixed norm, hierarchical norms

- Sparse Regression

- Simultaneous feature selection and regression

- Statistically consistent in the " $n<<p$ " regime

- Avoiding the Curse of Dimensionality

- Sparse regularizer with two considerations

- Not all locations are relevant

- Even if a location is relevant, not all variables are relevant 


\section{Sparse Group Lasso (SGL)}
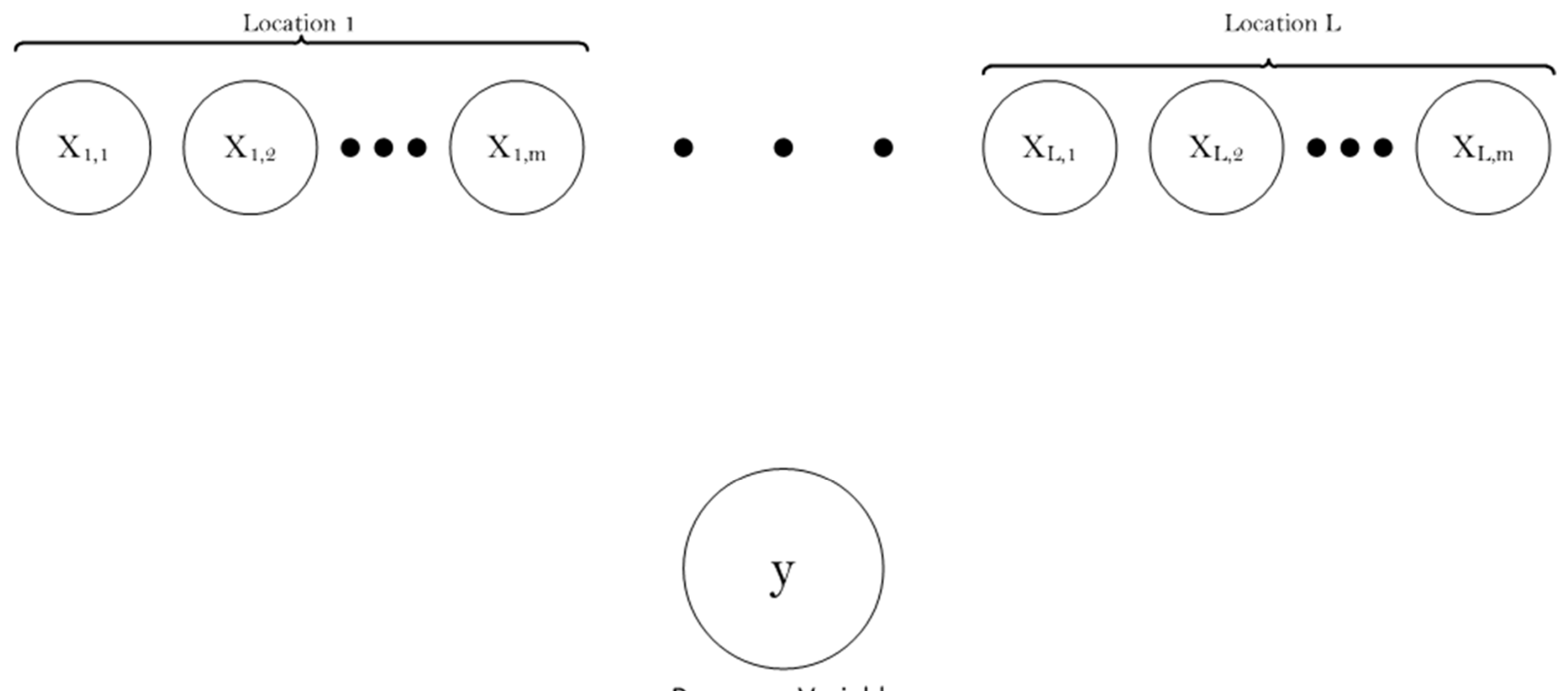

Response Variable 


\section{Sparse Group Lasso (SGL)}
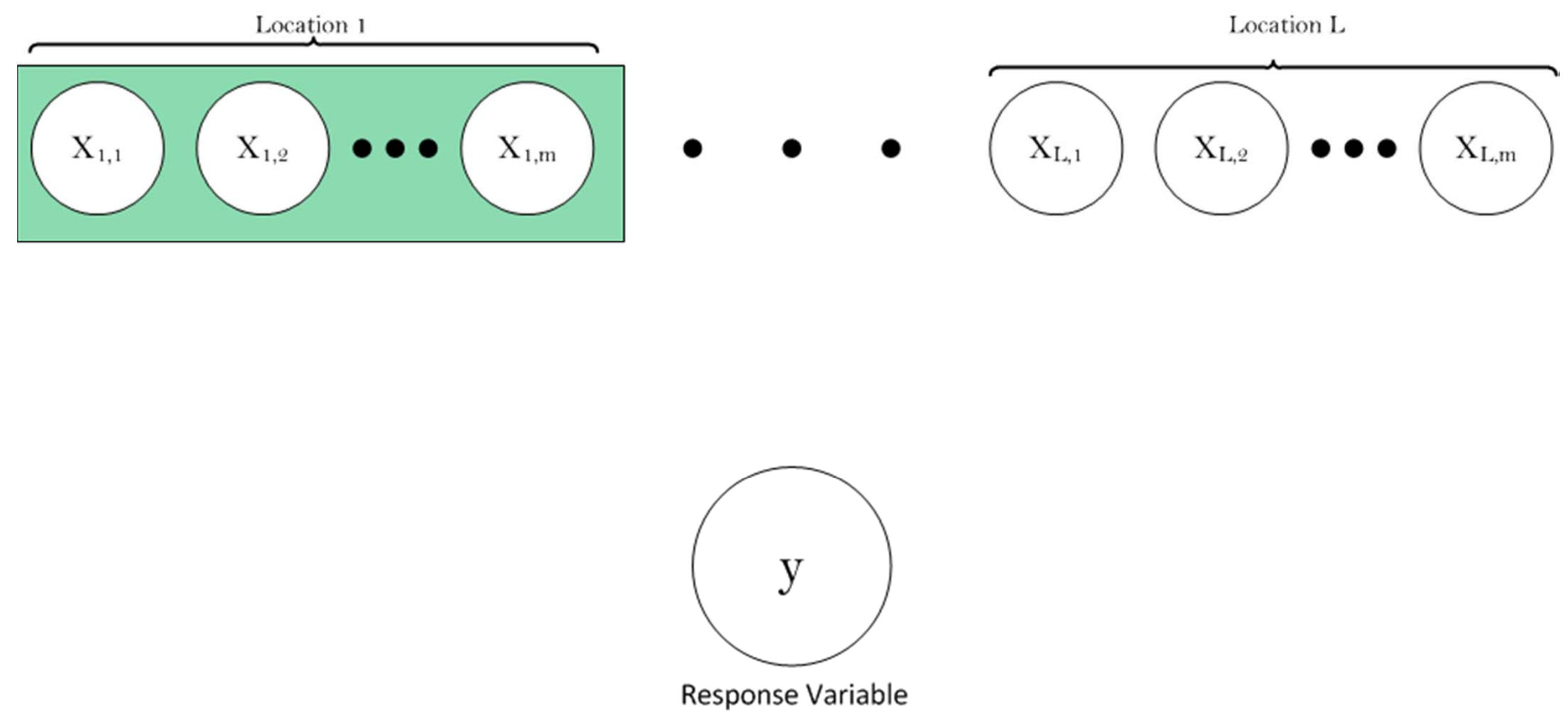


\section{Sparse Group Lasso (SGL)}

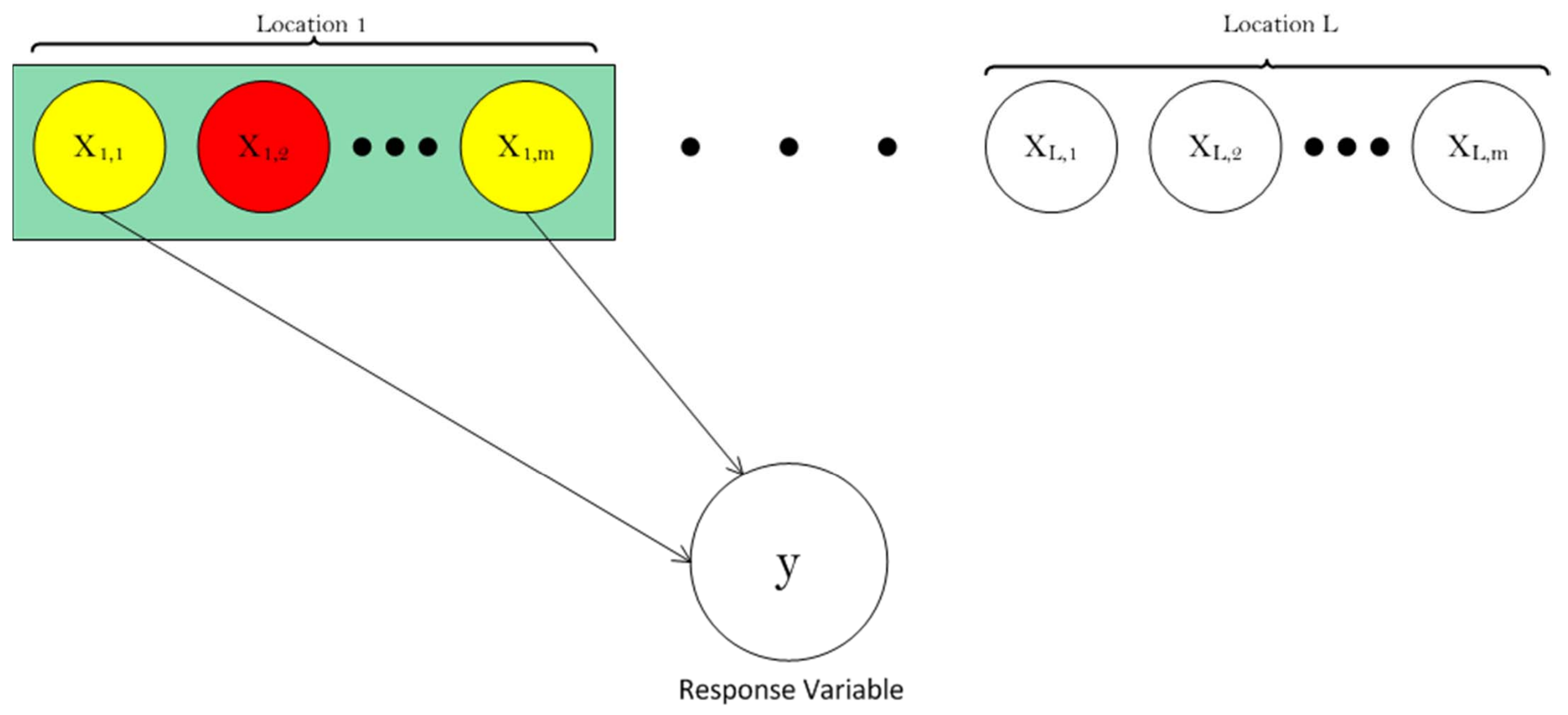




\section{Sparse Group Lasso (SGL)}

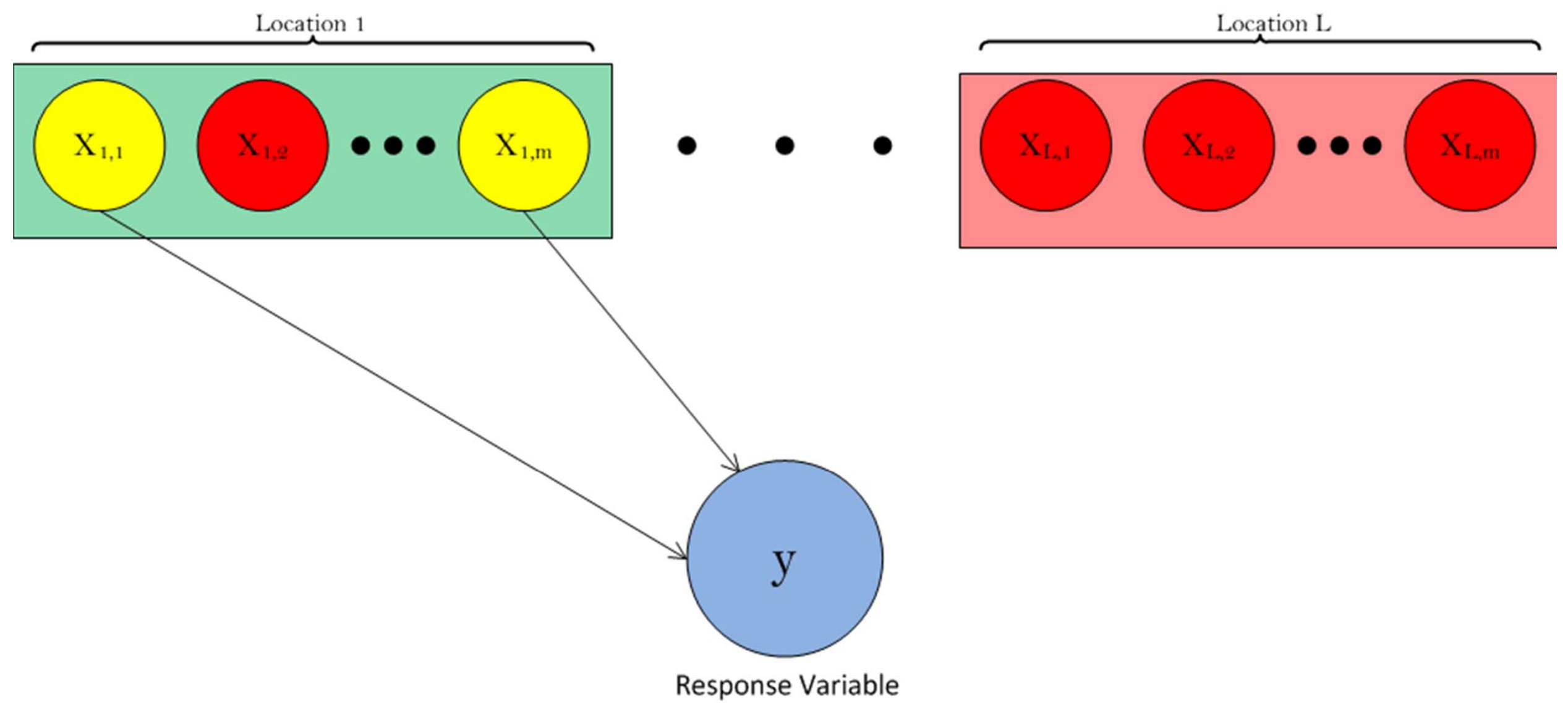




\section{Sparse Group Lasso (SGL)}

- High-dimensional Regression with "Sparse" "Group Sparsity"

$$
\begin{gathered}
\hat{\theta}_{S G L}=\underset{\theta \in \mathbb{R}^{m L}}{\operatorname{argmin}}\left\{\frac{1}{n}\|y-X \theta\|_{2}^{2}+\lambda_{1}\|\theta\|_{1}+\lambda_{2}\|\theta\|_{1, \mathcal{G}}\right\} \\
\|\theta\|_{1}=\sum_{i=1}^{m L}\left|\theta_{i}\right| \quad\|\theta\|_{1, \mathcal{G}}=\sum_{k=1}^{L}\left\|\theta_{G_{k}}\right\|_{2}
\end{gathered}
$$

$\mathcal{G}=\left\{G_{1}, \ldots, G_{L}\right\}:$ groups of $m$ variables at $L$ locations

- Efficient Optimization

- Primal: Proximal gradient method

- Dual: Block-coordinate dual ascent 


\section{Consistency of Sparse Group Lasso}

Theorem 1 Let $A$ be any subspace of $\mathbb{R}^{p}$ of dimension $s_{A}$. Let $\theta^{*}$ be the optimal (unknown) regression parameter, and let $r_{\left(1, \mathcal{G}_{2}, \alpha\right)}^{A^{\perp}}\left(\theta^{*}\right)$ be the sparse-group lasso norm of $\theta^{*}$ restricted to $A^{\perp}$, the orthogonal subspace of $A$. Then, if $\lambda_{n}$ satisfies the lower bound in Lemma 1, with probability at least $\left(1-\frac{2}{(p T)^{k}}\right)$, we have

$$
\left\|\hat{\theta}_{\lambda_{n}}-\theta^{*}\right\|_{2}^{2} \leq \frac{4 \lambda_{n}^{2}}{k_{\mathcal{L}}^{2}} s_{A}+\frac{2 \lambda_{n}}{k_{\mathcal{L}}} r_{\left(1, \mathcal{G}_{2}, \alpha\right)}^{A^{\perp}}\left(\theta^{*}\right)
$$

where $\hat{\theta}_{\lambda_{n}}$ is the SGL estimator.

Corollary 1 If the optimal parameter $\theta^{*}$ is in the subspade A, then

$$
\left\|\hat{\theta}_{\lambda_{n}}-\theta^{*}\right\|_{2}^{2} \leq \frac{4 \lambda_{n}^{2}}{k_{\mathcal{L}}^{2}} s_{A}=\left(O\left(\frac{\log p}{n}\right)\right) .
$$




\section{Prediction RMSE}

\begin{tabular}{|c|c|c|c|c|}
\hline Variable & Region & SGL & Network Clusters [4] & OLS \\
\hline \multirow{9}{*}{ 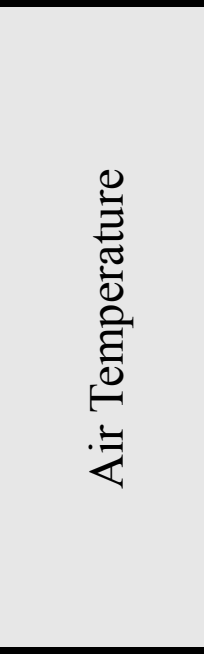 } & Brazil & 0.198 & 0.534 & 0.348 \\
\hline & Peru & 0.247 & 0.468 & 0.387 \\
\hline & West USA & 0.270 & 0.767 & 0.402 \\
\hline & East USA & 0.304 & 0.815 & 0.348 \\
\hline & W Europe & 0.379 & 0.936 & 0.493 \\
\hline & Sahel & 0.320 & 0.685 & 0.413 \\
\hline & S Africa & 0.136 & 0.726 & 0.267 \\
\hline & India & 0.205 & 0.649 & 0.300 \\
\hline & SE Asia & 0.298 & 0.541 & 0.383 \\
\hline \multirow{9}{*}{ 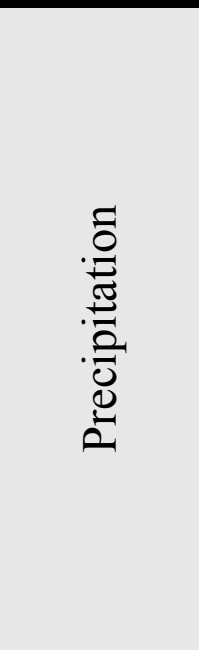 } & Brazil & 0.261 & 0.509 & 0.413 \\
\hline & Peru & 0.312 & 0.864 & 0.523 \\
\hline & West USA & 0.451 & 0.605 & 0.549 \\
\hline & East USA & 0.365 & 0.686 & 0.413 \\
\hline & W Europe & 0.358 & 0.45 & 0.551 \\
\hline & Sahel & 0.427 & 0.533 & 0.523 \\
\hline & S Africa & 0.235 & 0.697 & 0.378 \\
\hline & India & 0.146 & 0.672 & 0.264 \\
\hline & SE Asia & 0.159 & 0.665 & 0.312 \\
\hline
\end{tabular}




\section{SGL Regression: Brazil}

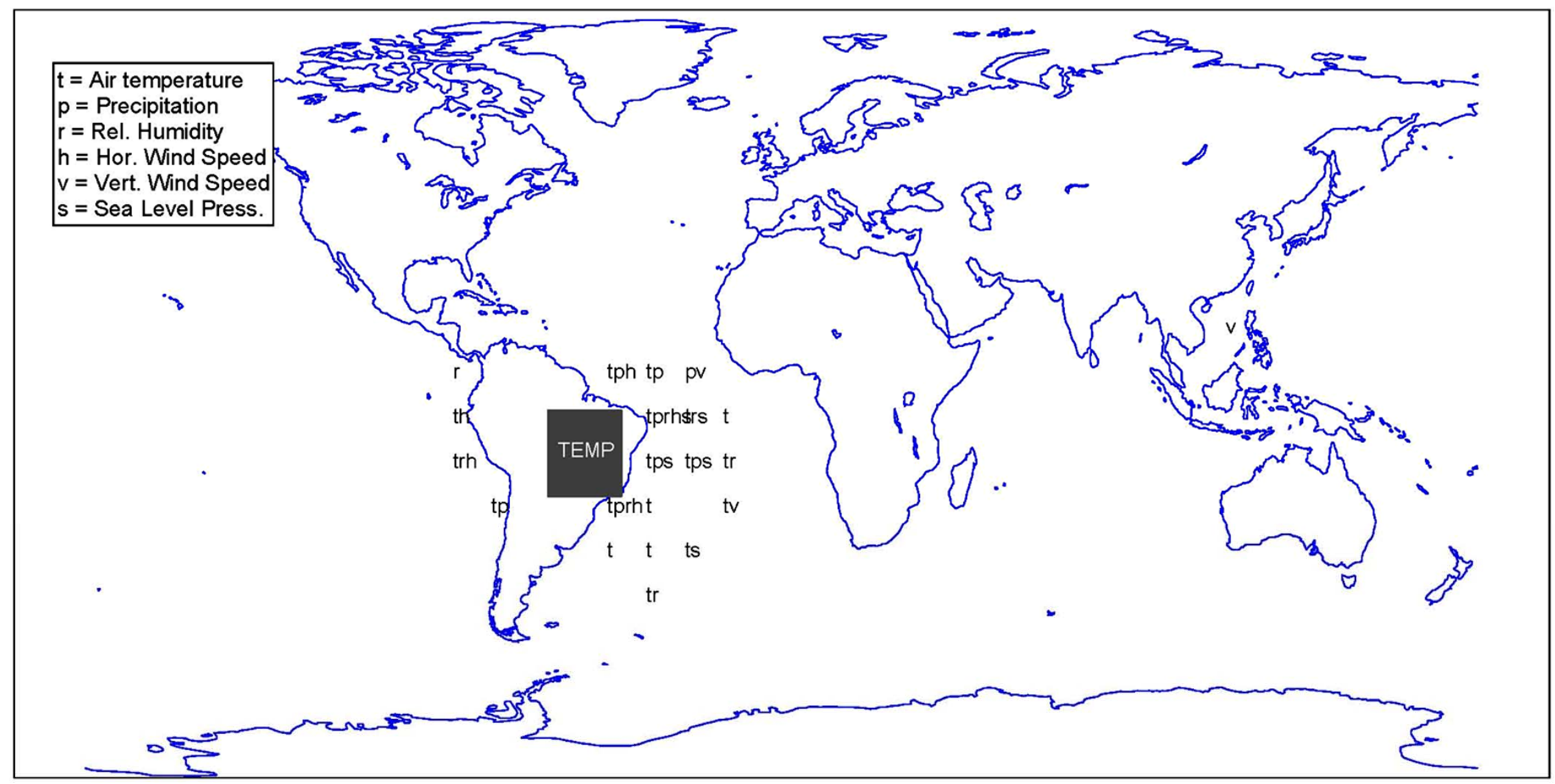

Relevant Covariates for Temperature in Brazil 


\section{SGL Regression: India}

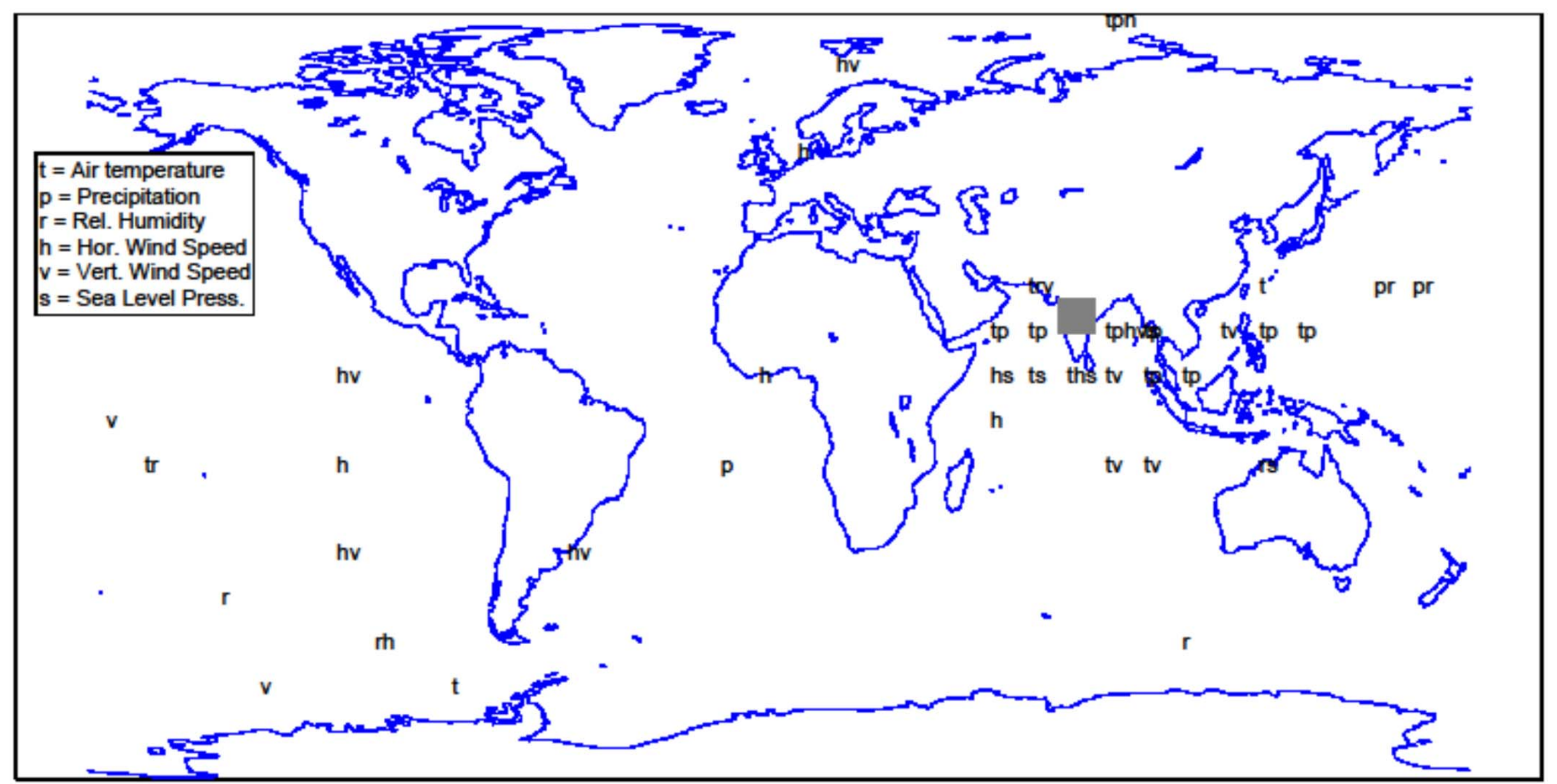

Relevant Covariates for Temperature in India 


\section{Statistical Networks}

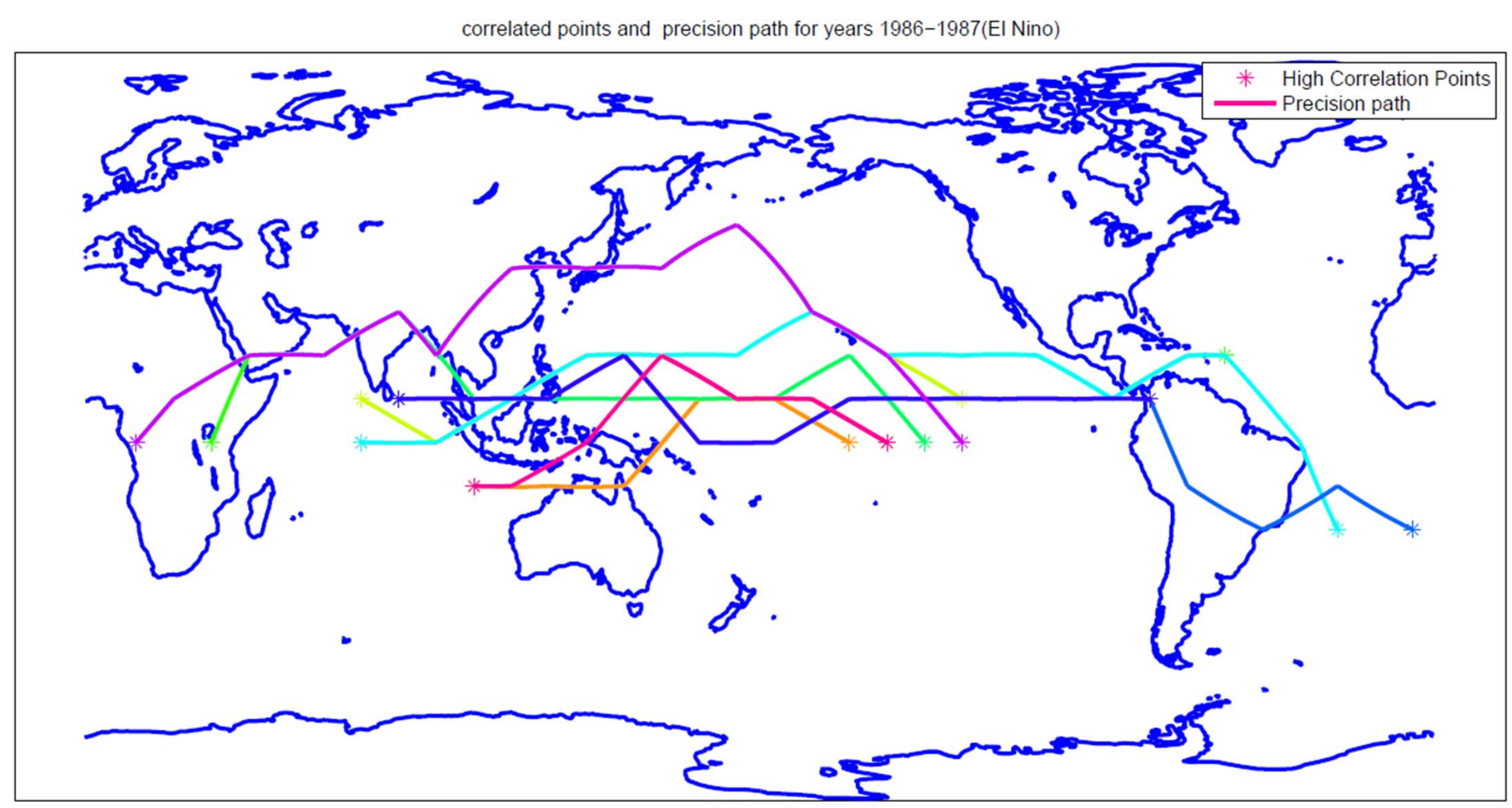

Graphical Models for Climate 


\section{Challenges and Open Questions}

- Abrupt Change Detection

- Detection: preprocessing, multivariate analysis, efficiency

- Attribution: multivariate, spatiotemporal, causal

- Abrupt Changes vs Extremes (intensity, duration, frequency)

- Sparse Regression, Statistical Networks

- Observable Setting

- Computing the precision matrix, Multivariate extensions

- Spatiotemporal dependencies, Oscillations

- Partially observable setting

- Problem formulation

- Latent variable models

- Tele-connections, Attributions

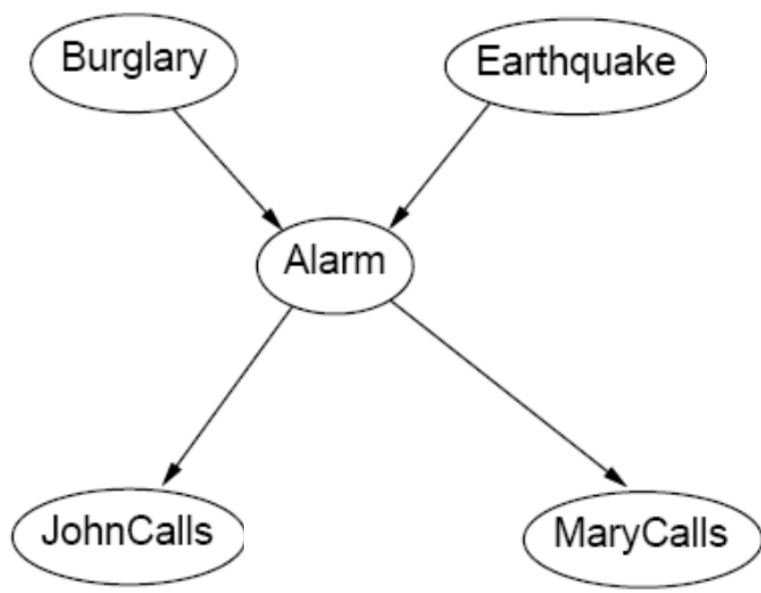




\section{Conclusions}

- Graphical Models

- Works with dependencies between variables

- Key problems: structure and parameter learning, inference

- Abrupt Change Detection

- Inference in discrete MRF

- High dimensional integer/linear program

- Detection of significant droughts from 1901-2006

- Sparse Regression and Networks

- Sparse group lasso for multivariate regression

- Statistical networks based on dependencies

- Key challenges

- Feynman's 3-step 'algorithm' for problem solving

- Questions+Expertise+Data from Climate Scientists 


\section{Acknowledgements}
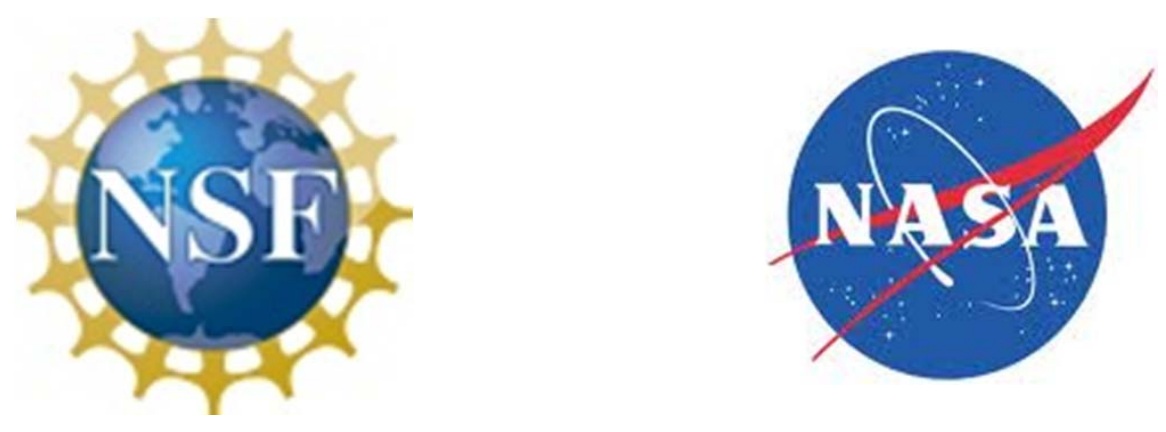

http://ucc.umn.edu

Soumyadeep Chatterjee, Snigdhansu Chatterjee, Qiang Fu, Auroop Ganguly, Stefan Liess, Peter Snyder, Karsten Steinhaeuser 


\section{a hank you,}

